\title{
Practice and Sociodemographic Factors Influencing Self- Monitoring of Blood Pressure in Ghanaians with Hypertension
}

\author{
Kennedy Dodam Konlan $\left(\mathbb{D}^{1},{ }^{1}\right.$ Charles Junior Afam-Adjei, ${ }^{2}$ Christian Afam-Adjei, $^{3}$ \\ Jennifer Oware, ${ }^{3}$ Theresa Akua Appiah, ${ }^{4}$ Kennedy Diema Konlan ${ }^{(D)}{ }^{5}$ \\ and Jeremiah Bella-Fiamawle ${ }^{2}$ \\ ${ }^{1}$ Department of Social \& Behavioural Sciences, School of Public Health, University of Ghana, Legon, Accra, Ghana \\ ${ }^{2}$ Department of Medicine, Nursing Directorate, Korle-Bu Teaching Hospital, Accra, Ghana \\ ${ }^{3}$ Department of Nursing, St. Karol School of Nursing, Aplaku-Accra, Ghana \\ ${ }^{4}$ School of Business, Ghana Institute of Management \& Public Administration (GIMPA), Accra, Ghana \\ ${ }^{5}$ Department of Public Health Nursing, School of Nursing and Midwifery, University of Allied Health Sciences, Ho, Ghana
}

Correspondence should be addressed to Kennedy Dodam Konlan; kennedy.konlan@gmail.com

Received 4 February 2020; Accepted 28 May 2020; Published 9 June 2020

Academic Editor: Khoa Nguyen

Copyright (C) 2020 Kennedy Dodam Konlan et al. This is an open access article distributed under the Creative Commons Attribution License, which permits unrestricted use, distribution, and reproduction in any medium, provided the original work is properly cited.

Background. In sub-Saharan Africa, the prevalence of hypertension has assumed epidemic levels and currently accounts for numerous complications such as stroke, heart failure, and kidney damage. Management of hypertension involves both drug and nonpharmacological approaches. Self-monitoring of blood pressure is an important nonpharmacological approach that facilitates early detection of deteriorating blood pressures and complications. Aims. We determined the practice and sociodemographic factors influencing self-monitoring of blood pressure among Ghanaians with hypertension. Methods. In a cross-sectional design, we recruited four hundred and forty-seven (447) Ghanaians with hypertension receiving care at the hypertensive Outpatient Department (OPD) Clinics of the Medical Department at the Korle-Bu Teaching Hospital (KBTH). The respondents were sampled using a simple random sampling technique of balloting without replacement. A structured questionnaire was used to gather data on the practice of self-monitoring of blood pressure and sociodemographic factors influencing self-monitoring in the respondents. We also measured some anthropometric and haemodynamic indices of the respondents. The data was entered in Microsoft Excel 2010 and exported into SPSS 21.0 to aid with the data analysis. A chisquare test and Student's $t$-test analysis were done to determine the relationship between the practice of self-monitioring and other sociodemographic variables. Data analayses were conducted at a significant level (alpha 0.05) and power of 95\% confidence. Thus, $p<0.05$ was considered statistically significant. Results. The practice of self-monitoring of blood pressure was $25.3 \%$ with more female respondents claiming to practice self-monitoring as compared to their male counterparts $(28.6 \%$ vs. 20.7\%). Awareness of self-monitoring of blood pressure was associated with increased practice of self-monitoring of blood pressure. Health workers (46.8\%), colleague patients (39.8\%), relatives/spouses (6.7\%), and the media (6.7\%) were identified as the sources of information about self-monitoring of blood pressure. Awareness of self-monitoring, level of education, valid health insurance, occupation, income levels, and marital status had a significant relationship with self-monitoring of blood pressure among the respondents. Thus, respondents with higher education, awareness of self-monitoring, valid health insurance, formal employment, and higher income were likely to monitor their blood pressure. Conclusion. Several sociodemographic factors influence the practice of self-monitoring of blood pressure in Ghanaians with hypertension. Thus, targeted hypertension education and social-cognitive interventions should focus on these sociodemographic factors so as to improve self-monitoring of blood pressure in order to reduce the complications of hypertension. 


\section{Introduction}

Hypertension, also known as high blood pressure (BP), is now a global public health problem associated with serious health complications [1]. Hypertension is a leading cause of global burden of heart diseases, stroke, renal failure, peripheral vascular disease, and premature mortality and disability $[1,2]$. The global burden of hypertension revealed that $25 \%$ of adults are living with hypertension and $9.2 \%$ of the total mortality is due to hypertension-related events [3]. It is estimated that over a billion people currently suffer from hypertension [1]. This figure is expected to increase to almost two billion in 2025 [1]. Recent studies from Ghana and other sub-Saharan African countries indicate that the prevalence of cardiovascular diseases (CVDs) is increasing at an alarming rate $[3,4]$.

The current management of hypertension involves pharmacological and nonpharmacological strategies $[4,5]$. The pharmacological approach involves using antihypertensive medications such as beta-blockers, beta-blockers with intrinsic sympathomimetic activity, alpha-1 blockers, combined alpha and beta-blockers, vasodilators, and angiotensin II receptor blocker $[5,6]$. Nonpharmacological strategies in the management of hypertension include dietary intake moderation or reduction (particularly saturated fats and excess sodium consumption), regular exercise, and avoidance of exposure to active and passive tobacco smoke [6]. Although significant efforts are being made by healthcare providers to control the morbidity and mortality associated with hypertension, it is still considered a major public health problem [7].

Proper management of hypertension requires adherence to management by patients as well as self-monitoring of blood pressure [8]. Self-monitoring of blood pressure refers to the measuring of one's own blood pressure (BP) outside the usual review visit to a general physician (GP), mostly within one's home $[2,6]$. It is the practice where a patient voluntarily measures his/her BP using a self-check device, be it manual or electronic $[7,9]$. Self-monitoring of blood pressure is crucial in detecting and preventing hypertension-related complications, and it has been shown to improve patient satisfaction and quality of life and also reduce primary care and out-patient and emergency department visits [10]. Most hypertensive patients in Ghana might not be aware of the enormous role of self-monitoring of blood pressure and hence may not practice efficient self-monitoring of blood pressure [11], thus depriving them of the enormous benefits associated with self-monitoring of blood pressure [9].

\section{Aim}

We determined the practice and sociodemographic factors influencing self-monitoring of blood pressure among Ghanaians with hypertension. In Ghana, complications and mortalities associated with hypertension are still high despite the marked improvement in care [4]. Self-monitoring of blood pressure has been established as one of the effective ways of reducing complications associated with hypertension [10]. Self-monitoring is also useful in getting clients with life- long conditions involved in their management and the surest way of patient empowerment $[9,11]$.

\section{Method}

3.1. Study Design. In this study, we employed a descriptive cross-sectional design. Ghanaians with hypertension receiving care at the Hypertensive Clinic of the Outpatient Department (OPD) of the Medical Department of the Korle-Bu Teaching Hospital (KBTH) were sampled. The philosophical underpinning of the study was the positivist strategy. In adopting the positivist approach to research, which is quantitative, the researcher embarks on the study of the reality by maintaining a distance between him/her and the researcher [12]. Contrary to this approach, researchers who hold the view of interpretivists (qualitative) adopt strategies which will lessen the distance between them and what is being studied [12]. In this study, our view was that self-monitoring of blood pressure and the sociodemographic determinants associated with it can be objectively measured hence the adoption of the positivist approach.

3.2. Setting. The study was conducted at the Korle-Bu Teaching Hospital (KBTH), a resource-constrained health facility in Ghana where there is consistent shortage of staff coupled with increasing patient numbers [13]. Thus, empowerment of patients with hypertension through self-monitoring of blood pressure could be useful in enhancing positive disease outcomes through early detection of uncontrolled blood pressures [10]. The KBTH is located in the southern part of Ghana, and it is the national referral hospital in Ghana. It is the third largest hospital in Africa [13]. The KBTH which was established in 1923 has grown from an initial 200 beds to 2000 beds and 17 clinical and diagnostic departments. The hospital is traditionally known to receive huge numbers of referral cases from across the country with daily average patient attendance of 1,500 with average daily admission of 250 patients [13]. The respondents were selected from the Hypertensive OPD Clinics of the Medical Department at the KBTH. These clinics run for four days in a week from $8 \mathrm{am}$ to $2 \mathrm{pm}$. The working days for the clinics were Mondays, Tuesdays, Thursdays, and Fridays. The data collection was done on Fridays.

3.3. Sample Size Determination. The sample size for the study was determined using the Cochran formula for determining the sample size [14]. Thus, the sample size of 385 respondents was required at a desired precision of 0.05 and at an estimated self-monitoring practice rate of 50\%. After adding $20 \%$ for nonresponse rate, the sample size for the study was estimated to be 461 respondents.

3.4. Selection of Participants and Data Collection. We included male and female hypertensive patients visiting the Hypertensive OPD Clinics of the Medical Department at the KBTH for review post discharge. The inclusion criteria for the selection of the study respondents included Ghanaian hypertensive patients between the ages of 35-65 years, a history of previous admission with hypertension, and being well oriented to time and place with no signs of mental illness. 
A simple random sampling technique of balloting without replacement was done using the patients' folders at the Hypertensive OPD Clinics of the Medical Department usually in the morning during waiting time. The patients' folders were numbered, and the corresponding numbers were written on small pieces of paper. The small pieces of paper with the numbers were then placed in a covered container and shaken thoroughly after which thirty (30) papers with corresponding numbers representing patients' folders were selected without replacement. The consent of those selected was obtained, and if they refused to consent, they were replaced by picking other small pieces of paper with the numbers corresponding to specific folders of patients until a total of thirty respondents were selected. The data collection was done on Fridays during a period of fifteen weeks (November, 2018, to March, 2019) excluding public holidays.

A pretested questionnaire was used as the data collection tool. Prior to the data collection, the questionnaire was pretested among hypertensive patients at the Polyclinic of the KBTH. The questionnaire contained sections which asked about the sociodemographic characteristics such as age of respondents, sex of respondents, highest educational level, marital status, occupation, income level, having a valid health insurance, awareness of normal blood pressure, and selfmonitoring, as well as the practice of self-monitoring of blood pressure. The data was entered into Microsoft Excel 2010. The data was then exported into the Statistical Package for Social Sciences (SPSS) version 21.0 to aid with the analysis.

3.5. Data Analysis. We analyzed the data using the Statistical Package for Social Sciences (SPSS) version 21.0. During the analysis, we did a gender comparison of sociodemographic variables using Pearson's $\chi^{2}$ test particularly for the dichotomous variables while using Student's $t$-test for a continuous variable (age). In determining the association between selfreported practice of self-monitoring of blood pressure and the sociodemographic variables, we used Pearson's $\chi^{2}$. In this study, $p<0.05$ was considered statistically significant.

3.6. Ethical Issues. The study data was part of data collected on a protocol titled: Adherence to Management and Quality of Life of Ghanaian Hypertensive on a Structured Health Education Programme (SHEP) approved by the Scientific and Technical Committee and the Institutional Review Board of the Korle-Bu Teaching Hospital (protocol identification number KBTH-IRB/0004/2018). We obtained permission from the Deputy Director of Nursing Service of the Central Outpatient Department prior to data collection. Also, we explained the purpose of the study to each respondent before obtaining written informed consent for the study. Further, we ensured confidentiality of the data collected by assigning unique codes to each response. We also maintained privacy and ensured anonymity throughout the data collection and analysis of the data.

\section{Results}

A total of four hundred and forty-seven (447) respondents took part in the study. As depicted in Table 1, the majority of the respondents (57.9\%) were females with $42.1 \%$ being males. The mean age of the respondents was $53.13 \pm 7.34$ years with the females older than the males $(54.13 \pm 8.46$ versus $51.21 \pm 4.83)$. The majority of the respondents $(61.3 \%)$ were married with only $4.3 \%, 11.6 \%$, and $22.6 \%$ being single, widowed, and divorced, respectively. The results revealed that the majority of the females (44.4\%) had no formal education with only $29(6.5 \%)$ of the respondents reporting having had tertiary education. Further, the study found that $46.5 \%$ reported as having low income level with $29.97 \%$ of the respondents claiming to have high income levels. Female respondents reported high income level as compared to their male counterparts $(41.3 \%$ versus $14.4 \%)$. Also, the results revealed that only $7.4 \%$ of the males had valid health insurance as compared to $63.7 \%$ of the females. The majority of the respondents did not have valid health insurance. Also, only $10.1 \%$ of the males had formal employment and this was similar among the females where $11.6 \%$ reported to have formal employment. With regard to unemployment, $41.3 \%$ of the females reported to be unemployed as compared to the $25.5 \%$ in males. The majority of the males were artisans/technicians or had vocational training (Table 1).

\section{Awareness and Source of Information about Self-Monitoring of Blood Pressure}

The majority (59.3\%) of the respondents said they were aware of their normal blood pressure even though most of them could not state the said normal blood pressure. Only, 194 (43.4\%) of the respondents said they were aware of self-monitoring of blood pressure with majority claiming not to be aware of self-monitoring. On the sources of information about self-monitoring of blood pressure, 90 (46.4\%) claimed health workers, 76 (39.2\%) cited colleague patients with only $10(5.7 \%)$, and $18(9.3 \%)$ cited spouses and media houses as sources of self-monitoring information (Table 2).

\section{Association between Practice of Self-Monitoring of Blood Pressure and Socio-Demographic Factors}

The practice of self-monitoring of blood pressure in the respondents was $25.3 \%$. Awareness of self-monitoring of blood pressure was associated with the practice of selfmotoring. The study revealed that higher educational qualification was associated with practice of self-monitoring of blood pressure. Respondents with formal employment were likely to self-monitor their blood pressure as compared to their counterparts. Also, having a valid health insurance was associated with the practice of self-monitoring. Further, a high income level was associated with self-monitoring of blood pressure. The results revealed that respondents who were single did more self-monitoring of their blood pressure as compared to married, widowed, and divorced counterparts (Table 3). 
TABLE 1: Sociodemographic characteristics of respondents.

\begin{tabular}{|c|c|c|c|}
\hline Variables & $\begin{array}{c}\text { Males, } n(\%) \\
188(42.1 \%)\end{array}$ & $\begin{array}{c}\text { Females, } n(\%) \\
259(57.9 \%)\end{array}$ & $\chi^{2}, p$ value \\
\hline Age (years) & $51.21 \pm 4.83$ & $54.13 \pm 8.46$ & $<0.001^{*}$ \\
\hline \multicolumn{4}{|l|}{ Marital status, n (\%) } \\
\hline Single & $18(9.6)$ & $1(0.4)$ & \multirow{4}{*}{$87.127,<0.001^{*}$} \\
\hline Married & $90(47.9)$ & $184(71)$ & \\
\hline Divorced & $73(38.9)$ & $28(10.8)$ & \\
\hline Widowed & $7(3.7)$ & $46(17.8)$ & \\
\hline \multicolumn{4}{|l|}{ Highest education, $\mathrm{n}(\%)$} \\
\hline No formal education & $90(47.9)$ & $115(44.4)$ & \multirow{5}{*}{$135.238,<0.001^{*}$} \\
\hline Junior secondary & $38(20.2)$ & $56(21.6)$ & \\
\hline Senior secondary & $22(11.7)$ & $37(14.3)$ & \\
\hline Vocational/technical & $32(17)$ & $32(12.4)$ & \\
\hline Tertiary & $6(3.2)$ & $19(7.3)$ & \\
\hline \multicolumn{4}{|l|}{ Income level, $\mathrm{n}(\%)$} \\
\hline High & $27(14.4)$ & $107(41.3)$ & \multirow{3}{*}{$74.478,<0.001^{*}$} \\
\hline Middle & $29(15.4)$ & $76(29.3)$ & \\
\hline Low & $132(70.2)$ & $76(29.3)$ & \\
\hline \multicolumn{4}{|l|}{ Valid health insurance } \\
\hline Yes & $14(7.4)$ & $165(63.7)$ & \multirow[t]{2}{*}{$143.606,<0.001^{*}$} \\
\hline No & $174(92.6)$ & $94(36.3)$ & \\
\hline \multicolumn{4}{|l|}{ Occupation } \\
\hline Formal employment & $19(10.1)$ & $30(11.6)$ & \multirow{5}{*}{$148.080,<0.001^{*}$} \\
\hline Retired & $1(0.5)$ & $30(11.6)$ & \\
\hline Trading/business & $2(1.1)$ & $60(23.2)$ & \\
\hline Artisan/technician/vocational & $118(62.8)$ & $32(12.4)$ & \\
\hline Unemployed & $48(25.5)$ & $107(41.3)$ & \\
\hline
\end{tabular}

* indicates statistical significance $(p<0.05)$.

TABle 2: Awareness and source of information about selfmonitoring of blood pressure.

\begin{tabular}{lcc}
\hline Variable & Frequency & Percentage \\
\hline Awareness of normal BP & & \\
$\quad$ Yes & 265 & 59.3 \\
$\quad$ No & 182 & 40.7 \\
Awareness of SM & & \\
$\quad$ Yes & 194 & 43.4 \\
No & 253 & 56.6 \\
Source of SM information & & \\
$\quad$ Media & 30 & 6.7 \\
Health workers & 209 & 46.8 \\
Relatives/spouse & 30 & 6.7 \\
Colleague patients & 178 & 39.8 \\
\hline
\end{tabular}

\section{Summary of Results}

The results as shown above indicate that the majority of hypertensive patients who seek care at the KBTH were females. Most of the respondents had low or no formal education with the majority under the low income brackets. The results indicate that the awareness of self-monitoring as well as the practice of self-monitoring of blood pressure was low even though awareness of normal blood pressure was reportedly high. The practice of self-monitoring of blood pressure was low and was associated with awareness of self-monitoring, educational level, valid health insurance, income level, and marital status.

\section{Discussion}

A total of 447 respondents were involved in the study, and the majority of the respondents were females indicating that more females sought health care rather than males. This finding is in line with the study done by $[14,15]$ who found out that more females sought health care as compared to males. On the awareness about self-monitoring, $43.4 \%$ of the respondents were aware of self-monitoring with the majority not being aware. Only $25.3 \%$ of the respondents claimed to be practicing self-monitoring of blood pressure indicating a low level of practice of self-monitoring. This finding is in line with the study by $[6,16]$ who found out that only $42 \%$ of patients among the respondents were measuring their blood pressure. This finding is in contrast with those found by [11, 15 ] where $88 \%$ of the patients were aware and agreed that 
TABLE 3: Comparing the association between practice of selfmonitoring of BP (SM) and sociodemographic factors in the respondents.

\begin{tabular}{|c|c|c|c|}
\hline Variable & $\begin{array}{l}\text { Total, } \\
N(\%)\end{array}$ & $\begin{array}{c}\text { Practice } \\
\text { of SM (\%) }\end{array}$ & $\chi^{2}, p$ value \\
\hline Awareness of SM & & & $49.240,<0.001^{*}$ \\
\hline Awareness of SM & $194(43.4)$ & $81(41.8)$ & \\
\hline Not aware of SM & $253(56.6)$ & $32(12.6)$ & \\
\hline Sex of respondent & & & $3.533,0.06$ \\
\hline Male & $188(42.1)$ & $39(20.7)$ & \\
\hline Female & $259(57.9)$ & $74(28.6)$ & \\
\hline Highest education & & & $253.560,<0.001^{*}$ \\
\hline No formal education & $205(45.9)$ & $14(6.8)$ & \\
\hline Junior high school & $94(21.0)$ & $11(11.7)$ & \\
\hline Senior high school & $59(13.2)$ & $8(13.6)$ & \\
\hline Vocational/technical & $64(14.3)$ & $10(15.6)$ & \\
\hline Tertiary & $25(5.6)$ & $8(32)$ & \\
\hline Valid health insurance & & & $32.707,<0.001^{*}$ \\
\hline Yes & $179(40)$ & $71(39.7)$ & \\
\hline No & $268(60)$ & $42(15.7)$ & \\
\hline Occupation & & & $256.298,<0.001^{*}$ \\
\hline Formal employment & $49(11)$ & $17(34.7)$ & \\
\hline Retired & $31(6.9)$ & $4(12.9)$ & \\
\hline Trading/business & $62(13.9)$ & $8(12.9)$ & \\
\hline Artisan/technician & $150(33.6)$ & $39(26)$ & \\
\hline Unemployed & $155(34.7)$ & $8(5.2)$ & \\
\hline Income level & & & $47.34,<0.001^{*}$ \\
\hline Low & $208(46.5)$ & $3(1.4)$ & \\
\hline Middle & $105(23.5)$ & $30(28.6)$ & \\
\hline High & $134(30)$ & $80(59.7)$ & \\
\hline Marital status & & & $45.397,<0.001^{*}$ \\
\hline Single & $19(4.3)$ & $14(73.7)$ & \\
\hline Married & $274(61.3)$ & $77(28.1)$ & \\
\hline Divorced & $101(22.6)$ & $6(5.9)$ & \\
\hline Widowed & $53(11.9)$ & $16(30.2)$ & \\
\hline
\end{tabular}

$*$ indicates statistical significance $(p<0.05)$.

self-monitoring of blood pressure was important and actually practiced it.

On the source of information about self-monitoring, health workers served as the main source of information. The media and relatives/spouses as a medium for information on self-monitoring were minimal. This result is in line with the study conducted by $[16,17]$. Also, $[18,19]$ in their study supported the view that the availability of information through family/friend/opinion leaders of trusted groups, the media (including radio, public enlightenment programmes, and newspapers), and the doctor/nurse/health worker can affect one's level of awareness on hypertension. Thus, health workers serve as a key source of information to patients, but the potential role of the media in health promotion is yet to be explored especially in developing countries. None of the respondents said they got their information from the internet despite the increasing internet service availability in Ghana. The need to explore the Internet as a viable means to deliver health educational messages was ought to be emphasized by health providers.

Also, the critical potential of family/spousal support in self-monitoring was found to be low as few of the respondents claimed their family members/spouses helped them with self-monitoring of their blood pressure or even made them aware of it. This finding is in contrast to the findings of [19-21] who observed that family, friends, and churches had powerful influences on patients' long-term management and adoption of healthy lifestyle practice. In a study by [20], 93\% of the hypertensive participants reported receiving social support from families while 55\% reported receiving social support from friends. Thus, family support remains a crucial need for hypertensive patients if complications of hypertension are to be reduced as they can encourage and support self-monitoring of blood pressure which can eventually reduce the risk of complications.

In our study, we found that the educational level of the respondents was found to be a significant determinant of practice of self-monitoring of blood pressure. The practice of self-monitoring increased marginally with the rising level of education among the respondents. Similar findings have been reported in other studies $[16,20]$ where the level of formal education was associated with self-monitoring practice in hypertensive patients.

The study found that the practice of self-monitoring of blood pressure increased with increasing self-reported income levels of respondents. Just as found in this study, other studies [16-18] report a positive relationship between the socioeconomic status of patients and the practice of self-monitoring of blood pressure. This could possibly be because of the fact that those within the high income brackets could afford to get blood pressure monitoring devices and thus could practice self-monitoring of their blood pressure at home without any difficulty as compared to their counterparts in the low-income brackets. The respondents in the lower income brackets probably had irregular clinic visits and did not have money to buy the blood pressure gadgets among others hence their low self-monitoring of blood pressure. Similar findings have been reported by some studies which stated that poor attendance to clinics and cost of blood pressure devices were identified by some researchers as a barrier to hypertension self-monitoring [20,21]. Chronic conditions like hypertension often result in physical disability such as stroke which may decrease one's strength and ability to work. This can reduce the income level of the patient and therefore serve as a source of financial constraint and hence a barrier to hypertensive self-monitoring $[15,17]$.

The study further found that those who had valid health insurance cards practiced self-monitoring of blood pressure as compared to their counterparts without valid health insurance. It is possible that those with valid health insurance were more likely to be regular in their clinic visitation; hence, they became aware of self-monitoring because it was taught at the OPD by nurses and doctors as compared to their counterparts without valid health insurance. Also, having valid health insurance could reduce the cost of the patient 
procuring their medications and hence providing alternative income to buy blood pressure devices for self-monitoring. According to $[6,17]$, resources are needed to support selfmonitoring and so a patient's socioeconomic position is of essence when it came to self-monitoring.

\section{Limitations of the Study}

The study used a cross-sectional design making it difficult to infer causation. Also, since the study depended on a pretested questionnaire which largely depended on the respondents' responses and recall, there is high possibility of recall bias. Further, income levels are usually associated with some social status in society; hence, some of the respondents could claim to be in the high social brackets when actually they are not and vice versa.

\section{Abbreviations \\ SM: Self-monitoring \\ CVDs: Cardiovascular diseases \\ BP: $\quad$ Blood pressure.}

\section{Data Availability}

The dataset supporting the conclusion of this study is available for systematic review and meta-analysis upon request.

\section{Conflicts of Interest}

The authors declare that they have no any competing/financial interests.

\section{Authors' Contributions}

KDK contributed to the conception, design, data analysis, and drafting of the manuscript and bears the primary responsibility for the content of the manuscript. CJAA revised the manuscript. CJA, JO, TAA, and JBF were involved in the data collection. KKD was involved in the revision of the manuscript. All the authors read and approved the content of the manuscript.

\section{Acknowledgments}

Our profound appreciation to the staff of the Training and Research Unit of the Nursing Directorate, Korle-Bu Teaching Hospital, who assisted during the field work.

\section{References}

[1] A. Moran, M. Forouzanfar, U. Sampson, S. Chugh, V. Feigin, and G. Mensah, "The epidemiology of cardiovascular diseases in sub-Saharan Africa: the global burden of diseases, injuries and risk factors 2010 study," Progress in Cardiovascular Diseases, vol. 56, no. 3, pp. 234-239, 2013.

[2] T. S. Ferguson, M. K. Tulloch-Reid, N. O. M. Younger et al., "Prevalence of the metabolic syndrome and its components in relation to socioeconomic status among Jamaican young adults: a cross-sectional study," BMC Public Health, vol. 10, no. 1 , p. $307,2010$.
[3] A. de-Graft Aikins, "Ghana's neglected chronic disease epidemic: a developmental challenge," Ghana Medical Journal, vol. 41, no. 4, pp. 154-159, 2007.

[4] S. H. Nyarko, "Prevalence and Sociodemographic Determinants of Hypertension History among Women in Reproductive Age in Ghana," International Journal of Hypertension, vol. 2016, Article ID 3292938, 6 pages, 2016.

[5] M. Seyedmazhari, "Pharmacological and non-pharmacological treatment of hypertension: a review article," ARYA Atherosclerosis, vol. 3, pp. S217-S221, 2013.

[6] F. Gohar, S. M. Greenfield, D. G. Beevers, G. Y. H. Lip, and K. Jolly, "Self-care and adherence to medication: a survey in the hypertension outpatient clinic," BMC Complementary and Alternative Medicine, vol. 8, no. 1, article 4, 2008.

[7] R. A. Adedoyin, C. E. Mbada, M. O. Balogun et al., "Prevalence and pattern of hypertension in a semi-urban community in Nigeria," European Journal of Cardiovascular Prevention \& Rehabilitation, vol. 15, no. 6, pp. 683-687, 2008.

[8] K. Yeboah, K. K. Dodam, P. K. Affrim et al., "Metabolic syndrome and parental history of cardiovascular disease in young adults in urban Ghana," BMC Public Health, vol. 18, no. 1, article 96, 2018.

[9] S. Baral-Grant, M. S. Haque, A. Nouwen, S. M. Greenfield, and R. J. McManus, "Self-Monitoring of Blood Pressure in Hypertension: A UK Primary Care Survey," International Journal of Hypertension, vol. 2012, Article ID 582068, 4 pages, 2012.

[10] D. Al Hadithi, A. S. Nazmi, and S. A. Khan, "Self monitoring of blood pressure (SMBP) among hypertensive patients in Muscat- a pilot study," Journal of Applied Pharmaceutical Science, vol. 2, no. 9, pp. 155-157, 2012.

[11] T. E. Ambakederemo, I. D. Ebuenyi, and J. Jumbo, "Knowledge and Attitude to Self-Monitoring Of Blood Pressure in a Cardiology Clinic in Nigeria," IOSR Journal of Dental and Medical Sciences, vol. 13, no. 5, pp. 63-65, 2014.

[12] J. W. Creswell, "Research design: qualitative, quantitative, and mixed methods approaches," in Research Design Qualitative Quantitative and Mixed Methods Approaches, p. 260, Sage Publications, 3rd edition, 2009.

[13] KBTH, Annual Report of KBTH, Korle Bu Teaching Hospital, Accra, 2016.

[14] L. G. Glynn, A. W. Murphy, S. M. Smith, K. Schroeder, and T. Fahey, "Interventions used to improve control of blood pressure in patients with hypertension," Cochrane Database Systematic Review, vol. 1, no. 3, article Cd005182, 2010.

[15] S. Drayton-Brooks and N. White, "Health promoting behaviors among African American women with faith-based support," Association of Black Nursing Faculty Journal., vol. 15, no. 5, pp. 84-90, 2014.

[16] V. Eugene and P. Bourne, "Hypertensive patients: knowledge, self-care management practices and challenges," Journal of Behavioral Health, vol. 2, no. 3, p. 1, 2012.

[17] M. Elkjaer, "E-health: Web-guided therapy and disease selfmanagement in ulcerative colitis. Impact on disease outcome, quality of life and compliance," Danish Medical Journal, vol. 59, no. 7, article B4478, 2012.

[18] E. Boulware, S. Flynn, J. Ameling et al., "Facilitators and barriers to hypertension self-management in urban African Americans: perspectives of patients and family members," Patient Preference and Adherence, vol. 7, pp. 741-749, 2013.

[19] A. Fex, G. Flensner, A. C. Ek, and O. Soderhamn, "Self-care agency and perceived health among people using advanced 
medical technology at home," Journal of Advanced Nursing, vol. 68, no. 4, pp. 806-815, 2012.

[20] B. Kaambwa, S. Bryan, S. Jowett et al., "Telemonitoring and self-management in the control of hypertension (TASMINH2): a cost-effectiveness analysis," European Journal Preventive Cardiology, vol. 21, no. 12, pp. 1517-1530, 2013.

[21] E. L. Knight, R. L. Bohn, P. S. Wang, R. J. Glynn, H. Mogun, and J. Avorn, "Predictors of uncontrolled hypertension in ambulatory patients," Hypertension, vol. 38, no. 4, pp. 809814, 2001. 